\title{
Comparison of efficacies of once-daily dose multimatrix mesalazine and multiple-dose mesalazine for the maintenance of remission in ulcerative colitis: a randomized, double-blind study
}

\author{
Haruhiko Ogata ${ }^{1}$, Akihiro Ohori ${ }^{2}$, Haruo Nishino $^{3}$, Seiichi Mizushima ${ }^{4}$, Atsushi Hagino ${ }^{4}$, Toshifumi Hibi ${ }^{5}$ \\ ${ }^{1}$ Center for Diagnostic and Therapeutic Endoscopy, Keio University Hospital, Tokyo, ${ }^{2}$ Inogashiradori Proctology and Gastroenterology Clinic, \\ Tokyo, ${ }^{3}$ Matsushima Clinic, Kanagawa, ${ }^{4}$ Clinical Development Department, Mochida Pharmaceutical Co., Ltd., Tokyo, ${ }^{5}$ Center for Advanced \\ IBD Research and Treatment, Kitasato Institute Hospital, Kitasato University, Tokyo, Japan
}

Background/Aims: This study compared the efficacy of once-daily administration of multimatrix mesalazine $2.4 \mathrm{~g} /$ day with multiple-dose mesalazine for the maintenance of remission. Methods: In this multicenter, randomized, double-blind study, 203 patients with ulcerative colitis in remission received multimatrix mesalazine $2.4 \mathrm{~g} /$ day once-daily or time-dependent (controlled-release) mesalazine $2.25 \mathrm{~g} /$ day 3 times-daily for 48 weeks. The primary efficacy endpoint was the proportion of patients without rectal bleeding. Results: The proportion of patients without rectal bleeding during the 48-week treatment period in the per protocol set was $84.8 \%(84 / 99)$ in the multimatrix mesalazine $2.4 \mathrm{~g}$ /day group and $78.0 \%(78 / 100)$ in the controlled-release mesalazine $2.25 \mathrm{~g}$ /day group. The difference between the 2 treatment groups was $6.8 \%$ (two-sided 95\% confidence interval, $-3.9 \%$ to $17.6 \%$ ). The noninferiority margin of $-10 \%$ was met in the comparison of multimatrix mesalazine $2.4 \mathrm{~g} /$ day once-daily with controlled-release mesalazine $2.25 \mathrm{~g} /$ day. Multimatrix mesalazine $2.4 \mathrm{~g} /$ day once-daily demonstrated consistent efficacy in all subgroups. There was no difference between the 2 treatment groups with regard to safety. Conclusions: A once-daily dose of 2 multimatrix mesalazine tablets $(2.4 \mathrm{~g})$ was not inferior to controlled-release mesalazine $2.25 \mathrm{~g} / \mathrm{day} 3$ times-daily in maintaining absence of rectal bleeding in ulcerative colitis. (Intest Res 2017;15:358-367)

Key Words: Colitis, ulcerative; Mesalazine; Maintenance; Once-daily

\section{INTRODUCTION}

Many placebo-controlled studies ${ }^{1-5}$ have been conducted to investigate the efficacy of mesalazine for the maintenance of remission in UC. However, only a small number of studies has directly compared the efficacy between different formulations. According to a Cochrane report, there are no differences in the efficacy or safety between the various formula-

Received October 10, 2016. Revised December 13, 2016. Accepted December 31, 2016. Published online February 22,2017

Correspondence to Haruhiko Ogata, Center for Diagnostic and Therapeutic Endoscopy, Keio University Hospital, 35 Shinanomachi, Shinjuku-ku, Tokyo 160-8582,

Japan. Tel: +81-3-3353-1211, Fax: +81-3-3353-3536, E-mail: hogata@z8.keio.jp

Financial support: The study was supported by Mochida Pharmaceutical Co., Ltd. Mochida Pharmaceutical Co., Ltd. provided funding to support the provision of multimatrix mesalazine (Shire US Inc.) and time-dependent controlled-release mesalazine (Kyorin Pharmaceutical Co., Ltd.). Conflict of interest: Haruhiko Ogata has received consulting, grant, or lecture fees from Mochida Pharmaceutical Co., Ltd., JIMRO, Takeda Pharmaceutical, Mitsubishi Tanabe Pharma, Kyorin Pharmaceutical, Otsuka Pharmaceutical, Astellas Pharma, Eisai, Zeria Pharmaceutical, AbbVie G.K., EA Pharma, and Boston Scientific Japan K.K. Akihiro Ohori has received lecture fees from Mitsubishi Tanabe Pharma and Zeria Pharmaceutical. Seiichi Mizushima and Atsushi Hagino are employees of Mochida Pharmaceutical Co., Ltd. Toshifumi Hibi is editor-in-chief of Intestinal Research and has received consulting, grant, lecture, or manuscript preparation fees from Mochida Pharmaceutical Co., Ltd., AbbVie G.K., EA Pharma, AstraZeneca K.K., JIMRO, Mitsubishi Tanabe Pharma, Eisai, Takeda Pharmaceutical, Zeria Pharmaceutical, Janssen Pharmaceutical K.K., Astellas Pharma, and Otsuka Pharmaceutical.

\footnotetext{
(c) Copyright 2017. Korean Association for the Study of Intestinal Diseases. All rights reserved.
}

This is an Open Access article distributed under the terms of the Creative Commons Attribution Non-Commercial License (http://creativecommons.org/licenses/by-nc/4.0)

which permits unrestricted non-commercial use, distribution, and reproduction in any medium, provided the original work is properly cited. 
tions of oral 5-aminosalicylic acid (5-ASA; mesalazine) in the maintenance of remission in UC. ${ }^{5}$

Multimatrix mesalazine (Lialda ${ }^{\circledR}$ in the United States; Mezavant ${ }^{\mathrm{TM}} \mathrm{XL}$ in the United Kingdom, Ireland, and Malta; Mezavant ${ }^{\mathrm{TM}}$ elsewhere in the European Union) is approved in Europe and the United States for once-daily (QD) use in the induction and maintenance of remission of mild-tomoderate UC. The approved dose for the maintenance of remission is $2.4 \mathrm{~g} /$ day QD, which is taken as 2 tablets per day. The efficacy of multimatrix mesalazine $2.4 \mathrm{~g} /$ day QD for maintenance of remission in UC was previously confirmed in 2 active-controlled studies. One study was conducted with Asacol $1.6 \mathrm{~g} /$ day twice-daily used as the comparator, and the primary endpoint was set as endoscopic remission (defined as a modified UC-Disease Activity Index (UC-DAI) endoscopic score of $\leq 1$ ) after 6 months of treatment. ${ }^{6}$ Asacol $1.6 \mathrm{~g} /$ day was the approved dose for the maintenance of remission of UC in the United States. The second study was conducted in patients with left-sided UC, with Asacol $2.4 \mathrm{~g}$ /day twice daily used the comparator. The co-primary endpoints were clinical remission (defined as a UC-DAI score of $\leq 1$ ) and clinical and endoscopic remission (endoscopic remission defined as a normal appearance upon endoscopic examination) after 12 months of treatment. ${ }^{7}$

This study investigated the efficacy and safety of 48 weeks of treatment with multimatrix mesalazine $2.4 \mathrm{~g} /$ day (Multimatrix-2.4 g/day) QD in patients with UC in remission, in comparison with time-dependent (controlled-release) mesalazine $2.25 \mathrm{~g} /$ day (Time- $2.25 \mathrm{~g} /$ day) 3 times-daily (TID) as the comparator.

\section{METHODS}

\section{Patients}

Eligible outpatients for the study fulfilled the following criteria: diagnosis of UC; age $\geq 16$ years at the time of informed consent; UC in remission, as defined by a UC-DAI score $\leq 2$ at randomization enrollment; and a rectal bleeding score of 0 . UC-DAI is a disease activity index of UC that consists of 4 variables: stool frequency, rectal bleeding, sigmoidoscopic findings of mucosal appearance, and physician's global assessment. Each variable is evaluated on a 4-point scoring scale from 0 to 3 , and the evaluation index is the total of the 4 scores (Table 1). ${ }^{8}$ Patients were excluded from the study for the following reasons: history of hypersensitivity to mesalazine and salicylic acid preparations; use of adrenal corticosteroids (oral, enema, suppository, medication for hemorrhoidal diseases, or injection) or cytapheresis therapy in the 2 weeks before randomization enrollment; received immune-regulating drug (oral, injection) or antitumor necrosis factor- $\alpha$ antibody in the 12 weeks before randomization enrollment; previous colonic resection (excluding appendectomy); moderate-to-severe renal or liver disorders; serious complications, including diseases of the blood, respiratory, gastrointestinal, or cardiovascular organs; diseases of the neuropsychiatric system; metabolic/electrolyte imbalance or hypersensitivity; or malignant tumor. The following groups were also excluded from the study: female patients who were pregnant, breastfeeding, possibly pregnant, or planning to get pregnant during the study period; and patients who had used other investigational products within the 4 months prior to enrollment into this study.

\section{Study Drugs}

Multimatrix mesalazine tablets (Lialda ${ }^{\circledR}$; Shire US Inc., Wayne, PA, USA) were red-brown, oval, film-coated tablets that contained $1.2 \mathrm{~g}$ of mesalazine per tablet. The comparator, controlled-release mesalazine tablets (Pentasa ${ }^{\circledR}$; Kyorin Pharmaceutical Co. Ltd., Tokyo, Japan), were white to paleyellow uncoated tablets with grayish-white to pale grayish-

Table 1. UC-Disease Activity Index

\begin{tabular}{|c|c|}
\hline Subscale & Score \\
\hline $\begin{array}{l}\text { Stool frequency } \\
\text { score }\end{array}$ & $\begin{array}{l}0 \text { : Normal (normal indicates healthy state or } \\
\text { maintained remission state of the subject) } \\
\text { 1: } 1-2 \text { Stools }>\text { normal } \\
2: 3-4 \text { Stools }>\text { normal } \\
3: \geq 5 \text { Stools }>\text { normal }\end{array}$ \\
\hline $\begin{array}{l}\text { Rectal bleeding } \\
\text { score }\end{array}$ & $\begin{array}{l}\text { 0: None } \\
\text { 1: Streaks of blood in stool } \\
\text { 2: Obvious blood in stool } \\
\text { 3: Mostly blood in stool }\end{array}$ \\
\hline $\begin{array}{l}\text { Sigmoidoscopy } \\
\text { score }\end{array}$ & $\begin{array}{l}\text { 0: Normal } \\
\text { 1: Mild (erythema, reduced vascular pattern, } \\
\quad \text { mild friability) } \\
\text { 2: Moderate (marked erythema, lack of vascular } \\
\text { pattern, friability, erosion) } \\
\text { 3: Severe (spontaneous bleeding, ulceration) }\end{array}$ \\
\hline PGA score & $\begin{array}{l}\text { 0: Normal } \\
\text { 1: Mild } \\
\text { 2: Moderate } \\
\text { 3: Severe }\end{array}$ \\
\hline
\end{tabular}

The mean score of daily stool frequency and rectal bleeding were calculated for the 3 days preceding each visit.

PGA, physician's global assessment. 
yellow maculae that contained $250 \mathrm{mg}$ of mesalazine per tablet. The study adopted a double-dummy trial design. The drug compliance for each study drug was measured based on the patient diary.

\section{Study Design}

This was a multicenter, randomized, double-blind, activecontrolled, parallel-group study with a 48-week treatment period that was conducted at 46 study centers in Japan from January 2011 to August 2012 (Japan Pharmaceutical Information Center clinical trial registration number: JapicCTI-101381). After informed consent was obtained, patient eligibility was evaluated based on the inclusion/exclusion criteria, and the study drug was administered to eligible patients. Patients were randomized to 1 of the 2 groups in a 1:1 ratio by the permuted block method, with each study center as 1 block. The 2 groups that received the study drugs were as follows: the Multimatrix- $2.4 \mathrm{~g} /$ day group and the Time$2.25 \mathrm{~g} /$ day group, and a double-dummy design was adopted to maintain blinding of the investigators and study participants. The Multimatrix-2.4 g/day group received 2 tablets of multimatrix mesalazine $1.2 \mathrm{~g}$ after breakfast QD and 3 tablets of controlled-release mesalazine placebo after each meal TID. The Time- $2.25 \mathrm{~g}$ /day group received 2 tablets of multimatrix mesalazine placebo after breakfast QD and 3 tablets of controlled-release mesalazine $250 \mathrm{mg}$ after each meal TID.

\section{Efficacy and Safety Evaluation}

During the study, patients were required to visit the study center every 4 weeks or at the time of discontinuation. At each visit, efficacy and safety were evaluated by the investigator. The mean scores of daily stool frequency and rectal bleeding were calculated for 3 days before each visit, based on the patient diary in which patients recorded stool frequency and rectal bleeding status. Colonoscopy was performed at the start of the treatment period and at week 48 or at the time of discontinuation, and the sigmoidoscopy score was evaluated by the same investigator with reference to the atlas of mucosal appearance. Each patient received an overall evaluation based on the clinical symptoms and endoscopic findings at the start of the treatment period and at week 48 or at the time of discontinuation, and the physician's global assessment score was evaluated.

Adverse events (AEs) were evaluated at each visit. Vital signs, body weight, and clinical laboratory tests were evalu- ated every 12 weeks or at the time of discontinuation. Clinical laboratory tests were also evaluated at week 4 . AEs were summarized by preferred terms of the Medical Dictionary for Regulatory Activities version 15.0 (http://www.meddra. $\operatorname{org} /)$.

\section{Objective/Endpoints}

The primary objective of the efficacy evaluation was to verify the noninferiority of Multimatrix-2.4 g/day QD to Time$2.25 \mathrm{~g} /$ day TID based on the primary efficacy endpoint, which was defined as the proportion of patients without rectal bleeding (rectal bleeding score of 0 ) during the treatment period. The primary objective of safety was to evaluate the treatment with Multimatrix-2.4 g/day QD and Time-2.25 g/ day TID in relation to AEs during the treatment period.

The secondary efficacy endpoints were: the duration of nonoccurrence of rectal bleeding (time to rectal bleeding score of $\geq 1$ during the treatment period); relapse (UC-DAI score $\geq 3$ and rectal bleeding score $\geq 1$ at the end of the treatment period); change in the UC-DAI score (UC-DAI score at the end of the treatment period-UC-DAI score at baseline); and the change in each variable of the UC-DAI (score at the end of the treatment period-score at baseline). The secondary safety endpoints were AEs (screening period, follow-up period) and adverse drug reactions (treatment period, follow-up period).

\section{Statistical Analysis}

The full analysis set (FAS) consisted of enrolled patients who received the study drug at least once and received a minimum of 1 efficacy evaluation. The per protocol set (PPS) was the primary analysis set for the efficacy analysis, which consisted of patients in the FAS that received an evaluation to ascertain the presence/absence of rectal bleeding after the start of the treatment period, satisfied the inclusion criteria and did not meet any exclusion criteria, did not receive prohibited concomitant drugs or therapies, and had a drug compliance of $\geq 75 \%$. The safety analysis set consisted of patients that received the study drug at least once and received a safety assessment.

The primary analysis for the efficacy was calculated from the proportion of patients without rectal bleeding (rectal bleeding score of 0 during the treatment period) and the two-sided 95\% CI of the difference between the treatment groups. The noninferiority margin $(\Delta)$ was set at $-10 \%$.

The secondary endpoint was calculated from the hazard 
ratio of the time to occurrence of rectal bleeding for the Multimatrix-2.4 g/day group compared with the Time- $2.25 \mathrm{~g} /$ day group and the two-sided 95\% CI were calculated. The proportion of patients with relapse and two-sided $95 \% \mathrm{CI}$ of the difference between treatment groups was calculated. The summary statistics of change in UC-DAI total score and individual variables were calculated. Subgroup analyses were carried out to support the primary endpoint. For the safety endpoint, the incidence of AEs was compared between the treatment groups.

The statistical analysis program SAS version 9.2 (SAS Institute, Cary, NC, USA) was used for calculations. With the assumption that the proportion of patients without rectal bleeding was $77 \%$ in Multimatrix-2.4 g/day group and $69 \%$ in Time-2.25 g/day group, we set the number of patients required to demonstrate the noninferiority to 95 patients per group at a one-sided type 1 error $(\alpha)$ of $2.5 \%, 80 \%$ power, and $\Delta=-10 \%$.

\section{Ethical Considerations}

The study was conducted in accordance with ethical principles that have their origin in the Declaration of Helsinki, the Ministerial Ordinance on Good Clinical Practice for Drugs, and other relevant laws, regulations, and standards.
Written informed consent was obtained from each patient. Prior to commencement of the study, the study protocol, a sample case report form, the patient information sheet, and informed consent form were approved by the Institutional Review Board at each study center; the appropriateness of the conduct of the clinical trial was also approved.

\section{RESULTS}

\section{Patient Disposition and Baseline Characteristics}

Consent was obtained from 220 patients. Seventeen patients withdrew before randomization and 203 received the study drugs (Multimatrix-2.4 g/day group, 100; Time$2.25 \mathrm{~g} /$ day group, 103). Of the 203 patients who received the study drugs, 165 patients completed the treatment of the study drugs and 38 patients (Multimatrix-2.4 g/day group, 17; Time-2.25 g/day group, 21) discontinued the study drugs during the treatment period (Fig. 1). In all treatment groups, the main reason for discontinuation was AEs, which included the aggravation of UC (Multimatrix-2.4 g/day group, 12; Time-2.25 g/day group, 11). The disposition of FAS and PPS is shown in Fig. 1. No patients were excluded from the FAS. Four patients were excluded from the PPS (Multimatrix-2.4 $\mathrm{g}$ /day group, 1; Time-2.25 g/day group, 3). The reasons for

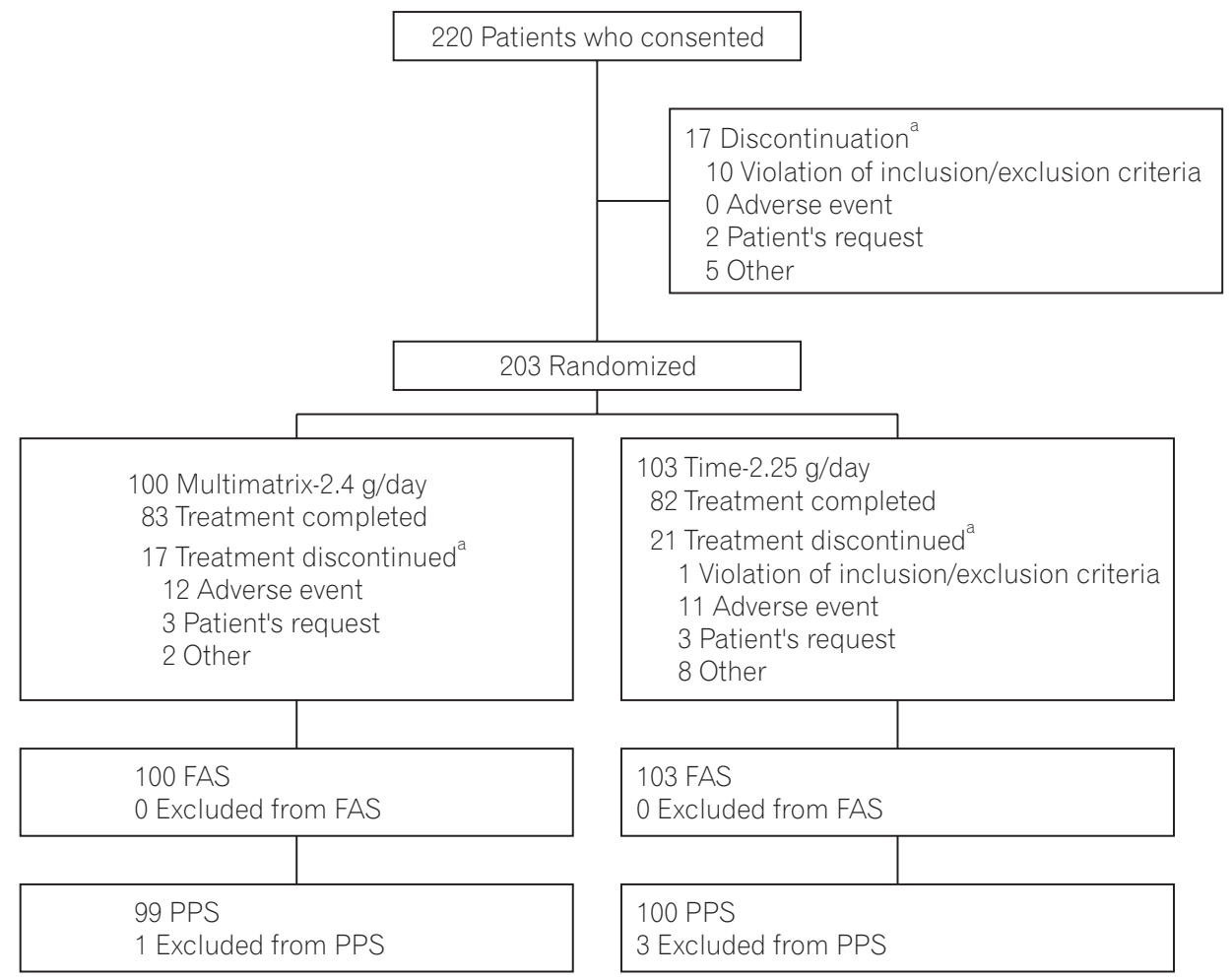

Fig. 1. Patient disposition. ${ }^{a}$ Multiple options are allowed as reasons for discontinuation. FAS, full analysis set; PPS, per protocol set. 
exclusion from the PPS were use of a prohibited concomitant drug, failing the inclusion criteria, and drug compliance of $<75 \%$. The mean rate of drug compliance in all analysis sets was not less than $95 \%$ in both treatment groups. There were no intergroup differences in patient background factors in the FAS (Table 2).

\section{Efficacy}

\section{1) Primary Endpoint}

In the PPS, the proportion of patients without rectal bleeding, the primary endpoint, was $84.8 \%$ (84/99) in the Multimatrix-2.4 g/day group and 78.0\% (78/100) in the Time-2.25 g/day group (Fig. 2). The difference between the 2 groups was $6.8 \%$ (two-sided $95 \% \mathrm{CI},-3.9 \%$ to $17.6 \%$ ). The lower limit of the two-sided $95 \%$ CI of the intergroup difference exceed-

Table 2. Patient Demographics (Full Analysis Set)

\begin{tabular}{|c|c|c|c|}
\hline Variable & Multimatrix-2.4 g/day $(n=100)$ & Time-2.25 g/day $(n=103)$ & $P$-value \\
\hline \multicolumn{4}{|l|}{ Sex } \\
\hline Male & $58(58.0)$ & $61(59.2)$ & $0.887^{\mathrm{a}}$ \\
\hline Female & $42(42.0)$ & $42(40.8)$ & - \\
\hline Age (yr) & $43.30 \pm 10.55$ & $44.50 \pm 12.68$ & $0.453^{b}$ \\
\hline $16-19$ & 0 & 0 & \\
\hline $20-29$ & $9(9.0)$ & $14(13.6)$ & \\
\hline $30-39$ & $33(33.0)$ & $23(22.3)$ & \\
\hline $40-49$ & $30(30.0)$ & $29(28.2)$ & \\
\hline $50-59$ & $19(19.0)$ & $23(22.3)$ & \\
\hline $60-64$ & $6(6.0)$ & $9(8.7)$ & \\
\hline$\geq 65$ & $3(3.0)$ & $5(4.9)$ & \\
\hline Height (cm) & $164.720 \pm 8.281$ & $164.840 \pm 8.673$ & $0.922^{b}$ \\
\hline Body weight (kg) & $60.410 \pm 11.030$ & $61.600 \pm 14.835$ & $0.516^{b}$ \\
\hline Disease course & & & $0.287^{\mathrm{a}}$ \\
\hline First attack & $18(18.0)$ & $15(14.6)$ & \\
\hline Relapsing-remitting & $82(82.0)$ & $85(82.5)$ & \\
\hline Chronic persistent & 0 & $3(2.9)$ & \\
\hline Extent of disease at the most recent flare-up & & & $0.483^{\mathrm{a}}$ \\
\hline Proctitis & $40(40.0)$ & $43(41.7)$ & \\
\hline Left-sided colitis & $33(33.0)$ & $27(26.2)$ & \\
\hline Pancolitis & $16(16.0)$ & $23(22.3)$ & \\
\hline Right-sided colitis & $1(1.0)$ & $3(2.9)$ & \\
\hline Segmental colitis & $3(3.0)$ & $4(3.9)$ & \\
\hline Unknown & $7(7.0)$ & $3(2.9)$ & \\
\hline UC-DAI score at baseline & $0.90 \pm 0.87$ & $0.80 \pm 0.83$ & $0.538^{b}$ \\
\hline Prior use of enema/suppository & & & - \\
\hline Mesalamine enema & 0 & $2(1.9)$ & \\
\hline Salazosulfapyridine suppository & 0 & $1(1.0)$ & \\
\hline
\end{tabular}

Values are presented as number (\%) or mean \pm SD.

${ }^{a}$ Fisher exact test.

${ }^{\mathrm{b}}$ t-test.

UC-DAI, UC-Disease Activity Index. 
ed the noninferiority margin $(-10 \%)$, thus demonstrating the noninferiority of Multimatrix-2.4 g/day QD to Time-2.25 g/ day TID. In the FAS, the proportion of patients without rectal bleeding was $85.0 \%(85 / 100)$ in the Multimatrix-2.4 g/day group and $78.6 \%(81 / 103)$ in the Time- $2.25 \mathrm{~g}$ /day group. The difference between the Multimatrix-2.4 $\mathrm{g}$ /day group and the Time- $2.25 \mathrm{~g} /$ day group was $6.4 \%$ (two-sided $95 \% \mathrm{CI},-4.2 \%$ to $16.9 \%$ ), which was comparable to the results from the PPS.

\section{2) Secondary Endpoints}

Fig. 3 shows the Kaplan-Meier plot for the duration of the nonoccurrence of rectal bleeding. Cox regression analysis was performed for the duration of the nonoccurrence of rectal bleeding and the result showed that the hazard ratio of

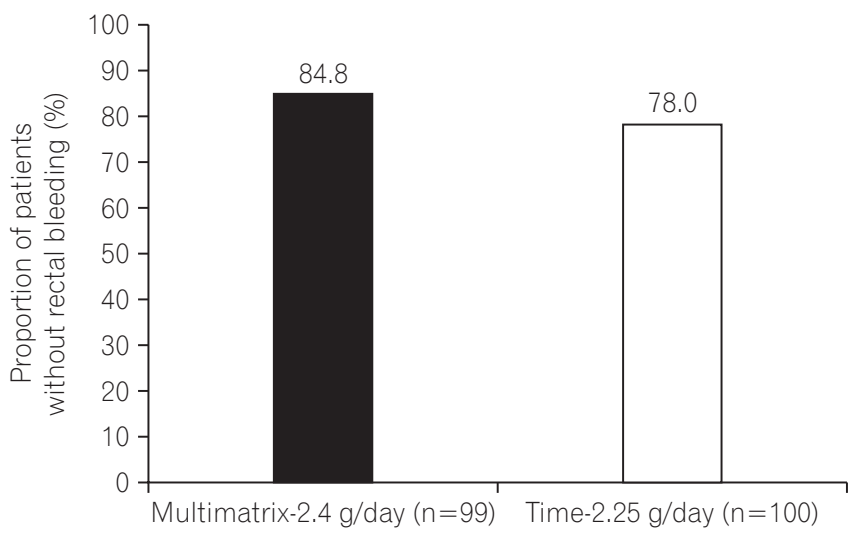

Fig. 2. The proportion of patients without rectal bleeding during the treatment period in the per protocol set. The difference between the multimatrix mesalazine $2.4 \mathrm{~g} /$ day group and the controlled-release mesalazine $2.25 \mathrm{~g} /$ day group was $6.8 \%$ (two-sided $95 \% \mathrm{Cl},-3.9 \%$ to $17.6 \%)$.

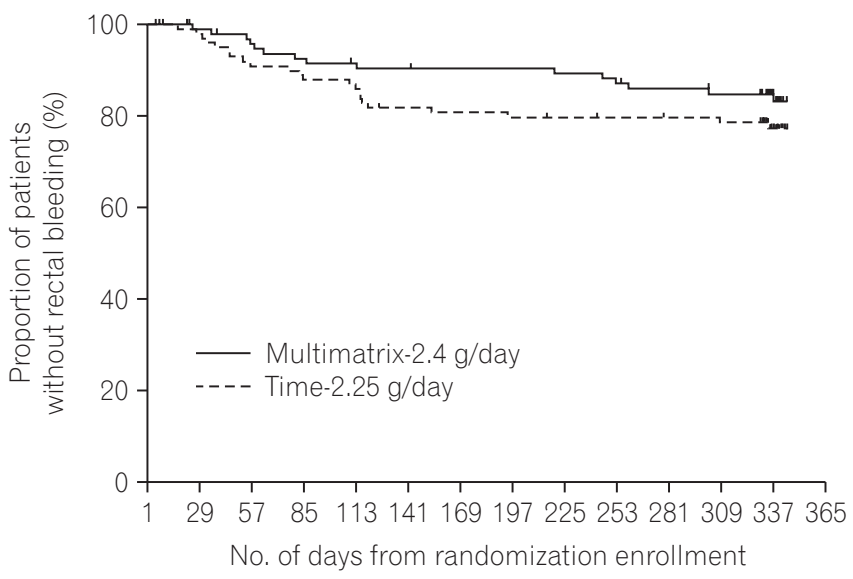

Fig. 3. The Kaplan-Meier plot of time to rectal bleeding (rectal bleeding score $\geq 1$ ) in the per protocol set. occurrence of rectal bleeding in the Multimatrix-2.4 g/day group compared with the Time- $2.25 \mathrm{~g} /$ day group was 0.677 (two-sided 95\% CI, 0.351-1.305). The risk of rectal bleeding occurring in the Multimatrix-2.4 g/day group decreased by $32.3 \%$ in comparison with that of the Time- $2.25 \mathrm{~g}$ /day group. The relapse rate at the end of treatment was $13.1 \%$ (13/99) in the Multimatrix-2.4 $\mathrm{g}$ /day group and $18.0 \%(18 / 100)$ in the Time-2.25 g/day group (Fig. 4). Table 3 shows the change in the UC-DAI score $(\operatorname{mean} \pm \mathrm{SD})$ at the end of treatment and the change in each subscore of the UC-DAI (mean $\pm \mathrm{SD})$. The results for secondary endpoints supported the results for the primary endpoint.

\section{3) Subgroup Analyses}

With regard to the main UC categories (classification by disease course, extent of disease, and UC-DAI score at baseline), the Multimatrix-2.4 g/day group showed consistent efficacy in all patient subgroups (Table 4).

\section{Safety}

The incidence of AEs during the treatment period was $82.0 \%(82 / 100)$ in the Multimatrix-2.4 $\mathrm{g} /$ day group and $85.4 \%$ (88/103) in the Time-2.25 g/day group (Table 5). The incidence of adverse drug reactions (side effects) during the treatment period was $17.0 \%$ (17/100) in the Multimatrix-2.4 $\mathrm{g}$ /day group and $25.2 \%(26 / 103)$ in the Time-2.25 g/day group. The frequency, type, and time to onset of AEs and

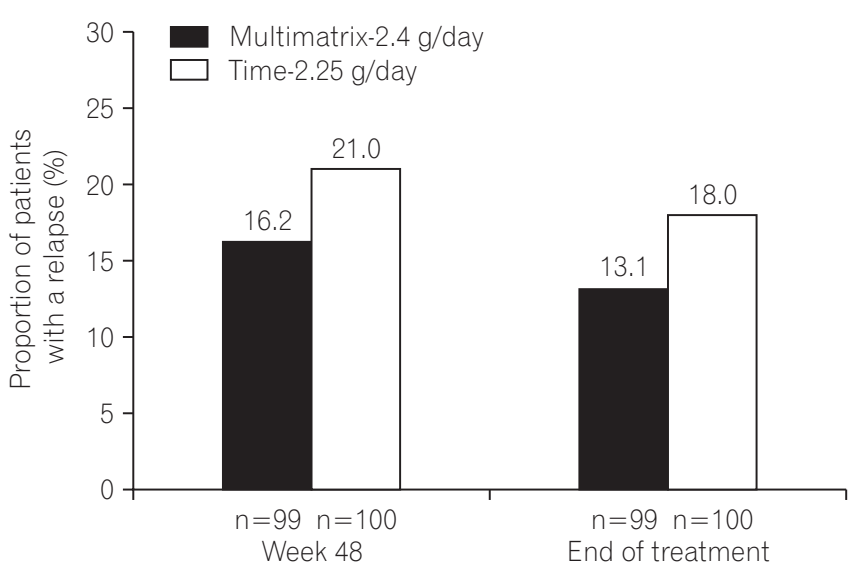

Fig. 4. The proportion of patients who relapsed (UC-Disease Activity Index score $\geq 3$ and rectal bleeding score $\geq 1$ ) at week 48 or at the discontinuation of treatment in the per protocol set. The difference between the multimatrix mesalazine $2.4 \mathrm{~g} /$ day group and the controlled-release mesalazine $2.25 \mathrm{~g} /$ day group was $-4.8 \%$ (two-sided $95 \% \mathrm{Cl}_{1}-15.6 \%$ to $5.9 \%$ ) at week 48 and $-4.9 \%$ (two-sided $95 \% \mathrm{Cl}_{1}-14.9 \%$ to $5.2 \%$ ) at the end of treatment period. 
Table 3. Change in UC-DAl Score at End of Treatment (Per Protocol Set)

\begin{tabular}{|c|c|c|}
\hline Variable & $\begin{array}{c}\text { Multimatrix-2.4 } \\
\text { g/day }\end{array}$ & $\begin{array}{l}\text { Time-2.25 } \\
\text { g/day }\end{array}$ \\
\hline \multicolumn{3}{|l|}{ UC-DAI score } \\
\hline No. of patients & 92 & 95 \\
\hline Mean $\pm S D$ & $0.50 \pm 1.94$ & $0.60 \pm 2.02$ \\
\hline $\begin{array}{l}\text { Difference between groups } \\
(95 \% \mathrm{Cl})\end{array}$ & $-0.1(-0.7$ to 0.5$)$ & - \\
\hline \multicolumn{3}{|l|}{ Stool frequency score } \\
\hline No. of patients & 98 & 100 \\
\hline Mean $\pm S D$ & $0.10 \pm 0.68$ & $0.20 \pm 0.58$ \\
\hline $\begin{array}{l}\text { Difference between groups } \\
(95 \% \mathrm{Cl})\end{array}$ & $0.0(-0.2$ to 0.2$)$ & - \\
\hline \multicolumn{3}{|l|}{ Rectal bleeding score } \\
\hline No. of patients & 98 & 100 \\
\hline Mean $\pm S D$ & $0.10 \pm 0.56$ & $0.20 \pm 0.51$ \\
\hline $\begin{array}{l}\text { Difference between groups } \\
(95 \% \mathrm{Cl})\end{array}$ & $0.0(-0.2$ to 0.1$)$ & - \\
\hline \multicolumn{3}{|l|}{ Sigmoidoscopy score } \\
\hline No. of patients & 92 & 95 \\
\hline Mean $\pm S D$ & $0.10 \pm 0.65$ & $0.10 \pm 0.79$ \\
\hline $\begin{array}{l}\text { Difference between groups }{ }^{\mathrm{a}} \\
(95 \% \mathrm{Cl})\end{array}$ & $0.0(-0.2$ to 0.2$)$ & - \\
\hline \multicolumn{3}{|c|}{ Physician's global assessment score } \\
\hline No. of patients & 92 & 95 \\
\hline Mean $\pm S D$ & $0.10 \pm 0.57$ & $0.10 \pm 0.62$ \\
\hline $\begin{array}{l}\text { Difference between groups } \\
(95 \% \mathrm{Cl})\end{array}$ & $0.0(-0.2$ to 0.2$)$ & - \\
\hline
\end{tabular}

Change in each score=score at the end of treatment-score at baseline. a Differences in mean change of scores between groups, adjusted according to score at baseline (Multimatrix-2.4 g/day-Time-2.25 g/day). UC-DAl, UC-Disease Activity Index.

adverse drug reactions during the treatment period in each treatment group were comparable. In the Multimatrix-2.4 $\mathrm{g}$ /day group, there was no increase in the incidence of AEs nor adverse drug reactions correlated with the length of the treatment period. There were no deaths. The following serious AEs occurred during the treatment period: cervix carcinoma ( 1 patient) and aggravation of UC ( 1 patient) in the Multimatrix-2.4 g/day group, and ovarian cyst (1 patient) in the Time- $2.25 \mathrm{~g} /$ day group. No causal relationship was found with the study drugs.
Table 4. Proportion of Patients without Rectal Bleeding by Subgroup (Per Protocol Set)

\begin{tabular}{|c|c|c|}
\hline Variable & $\begin{array}{c}\text { Multimatrix-2.4 } \\
\text { g/day }\end{array}$ & $\begin{array}{c}\text { Time-2.25 } \\
\text { g/day }\end{array}$ \\
\hline \multicolumn{3}{|l|}{ Disease course } \\
\hline \multicolumn{3}{|l|}{ First attack } \\
\hline No. of patients & 18 & 15 \\
\hline $\begin{array}{l}\text { No. of patients without rectal } \\
\text { bleeding }(\%)\end{array}$ & 15 (83.3) & $12(80.0)$ \\
\hline $\begin{array}{l}\text { Differences between groups }{ }^{\mathrm{a}} \\
(95 \% \mathrm{Cl})\end{array}$ & $3.3(-23.2$ to 29.9$)$ & - \\
\hline Relapse-remitting & - & \\
\hline No. of patients & 81 & 82 \\
\hline $\begin{array}{l}\text { No. of patients without rectal } \\
\text { bleeding }(\%)\end{array}$ & $69(85.2)$ & $63(76.8)$ \\
\hline $\begin{array}{l}\text { Differences between groups }{ }^{\mathrm{a}} \\
(95 \% \mathrm{Cl})\end{array}$ & $8.4(-3.6$ to 20.3$)$ & - \\
\hline \multicolumn{3}{|l|}{ Extent of disease } \\
\hline \multicolumn{3}{|l|}{ Proctitis } \\
\hline No. of patients & 40 & 43 \\
\hline $\begin{array}{l}\text { No. of patients without rectal } \\
\text { bleeding }(\%)\end{array}$ & 33 (82.5) & $32(74.4)$ \\
\hline $\begin{array}{l}\text { Differences between groups }{ }^{a} \\
(95 \% \mathrm{Cl})\end{array}$ & 8.1 (-9.5 to 25.7$)$ & - \\
\hline \multicolumn{3}{|l|}{ Left-sided colitis } \\
\hline No. of patients & 32 & 26 \\
\hline $\begin{array}{l}\text { No. of patients without rectal } \\
\text { bleeding }(\%)\end{array}$ & $28(87.5)$ & $19(73.1)$ \\
\hline $\begin{array}{l}\text { Differences between groups } \\
(95 \% \mathrm{Cl})\end{array}$ & 14.4 (-6.1 to 35.0$)$ & - \\
\hline \multicolumn{3}{|l|}{ Pancolitis } \\
\hline No. of patients & 16 & 21 \\
\hline $\begin{array}{l}\text { No. of patients without rectal } \\
\text { bleeding }(\%)\end{array}$ & $14(87.5)$ & $19(90.5)$ \\
\hline $\begin{array}{l}\text { Differences between groups }{ }^{\mathrm{a}} \\
(95 \% \mathrm{Cl})\end{array}$ & $-3.0(-23.5$ to 17.5$)$ & - \\
\hline \multicolumn{3}{|l|}{ Right-sided colitis } \\
\hline No. of patients & 1 & 3 \\
\hline $\begin{array}{l}\text { No. of patients without rectal } \\
\text { bleeding }(\%)\end{array}$ & $1(100.0)$ & $2(66.7)$ \\
\hline $\begin{array}{l}\text { Differences between groups }{ }^{a} \\
(95 \% \mathrm{Cl})\end{array}$ & $33.3(-20.0$ to 86.7$)$ & - \\
\hline \multicolumn{3}{|l|}{ Segmental colitis } \\
\hline No. of patients & 3 & 4 \\
\hline $\begin{array}{l}\text { No. of patients without rectal } \\
\text { bleeding }(\%)\end{array}$ & $3(100.0)$ & $3(75.0)$ \\
\hline $\begin{array}{l}\text { Differences between groups } \\
(95 \% \mathrm{Cl})\end{array}$ & $25.0(-17.4$ to 67.4$)$ & - \\
\hline
\end{tabular}


Table 4. Continued

\begin{tabular}{|c|c|c|}
\hline Variable & $\begin{array}{c}\text { Multimatrix-2.4 } \\
\text { g/day }\end{array}$ & $\begin{array}{c}\text { Time-2.25 } \\
\text { g/day }\end{array}$ \\
\hline \multicolumn{3}{|l|}{ Unknown } \\
\hline No. of patients & 7 & 3 \\
\hline $\begin{array}{l}\text { No. of patients without rectal } \\
\text { bleeding }(\%)\end{array}$ & $5(71.4)$ & $3(100.0)$ \\
\hline $\begin{array}{l}\text { Differences between groups }{ }^{\mathrm{a}} \\
(95 \% \mathrm{Cl})\end{array}$ & $-28.6(-62.0$ to 4.9$)$ & - \\
\hline \multicolumn{3}{|l|}{ UC-DAI score at baseline } \\
\hline \multicolumn{3}{|l|}{0} \\
\hline No. of patients & 45 & 47 \\
\hline $\begin{array}{l}\text { No. of patients without rectal } \\
\text { bleeding }(\%)\end{array}$ & 41 (91.1) & 38 (80.9) \\
\hline $\begin{array}{l}\text { Differences between groups } \\
(95 \% \mathrm{Cl})\end{array}$ & $10.3(-3.7$ to 24.2$)$ & - \\
\hline \multicolumn{3}{|l|}{1} \\
\hline No. of patients & 23 & 28 \\
\hline $\begin{array}{l}\text { No. of patients without rectal } \\
\text { bleeding }(\%)\end{array}$ & $20(87.0)$ & $22(78.6)$ \\
\hline $\begin{array}{l}\text { Differences between groups } \\
(95 \% \mathrm{Cl})\end{array}$ & $8.4(-12.1$ to 28.9$)$ & - \\
\hline \multicolumn{3}{|l|}{2} \\
\hline No. of patients & 31 & 25 \\
\hline $\begin{array}{l}\text { No. of patients without rectal } \\
\text { bleeding }(\%)\end{array}$ & $23(74.2)$ & $18(72.0)$ \\
\hline $\begin{array}{l}\text { Differences between groups }{ }^{\mathrm{a}} \\
(95 \% \mathrm{Cl})\end{array}$ & $2.2(-21.2$ to 25.6$)$ & - \\
\hline
\end{tabular}

Change in UC-DAI score=UC-DAI score at the end of treatment-UCDAl score at baseline.

aDifferences in mean change of scores between groups, adjusted according to score at baseline (Multimatrix-2.4 g/day-Time-2.25 g/day). UC-DAI, UC-Disease Activity Index.

\section{DISCUSSION}

The efficacy of QD dosing compared with that of multiple dosing of the same formulation of mesalazine has been investigated in several previous studies. A double-blind, double-dummy study of controlled-release mesalazine demonstrated the noninferiority of QD to TID after 52 weeks of administration at the dose of $1.5 \mathrm{~g} /$ day to $2.25 \mathrm{~g} /$ day. ${ }^{9}$ Another randomized, single-blind, phase III study of $\mathrm{pH}$-dependent delayed-release mesalazine $1.6 \mathrm{~g} /$ day to $2.4 \mathrm{~g} /$ day showed that QD dosing was noninferior to twice-daily dosing in the maintenance of clinical remission for 6 months. ${ }^{10}$ A randomized, single-blind, phase III study of controlled-release
Table 5. Incidence of Adverse Events

\begin{tabular}{lcc}
\hline \multicolumn{1}{c}{ Characteristic } & $\begin{array}{c}\text { Multimatrix-2.4 } \\
\text { g/day }(\mathbf{n = 1 0 0 )}\end{array}$ & $\begin{array}{c}\text { Time-2.25 } \\
\mathbf{g} / \text { day }(\mathbf{n = 1 0 3 )}\end{array}$ \\
\hline Total & $82(82.0)$ & $88(85.4)$ \\
Nasopharyngitis & $41(41.0)$ & $48(46.6)$ \\
Aggravation of UC & $10(10.0)$ & $12(11.7)$ \\
Diarrhea & $5(5.0)$ & $5(4.9)$ \\
Headache & $4(4.0)$ & $10(9.7)$ \\
N-acetyl- $\beta$-D- & $3(3.0)$ & $9(8.7)$ \\
glucosaminidase increase & & \\
Gastroenteritis & $3(3.0)$ & $6(5.8)$ \\
Gastritis & $2(2.0)$ & $7(6.8)$ \\
Back pain & $2(2.0)$ & $6(5.8)$ \\
\hline
\end{tabular}

Values are presented as number (\%). Adverse events reported by at least $5 \%$ of subjects in any treatment group during the treatment period are listed.

mesalazine reported that the proportion of patients that maintained remission after 12 months of treatment with QD dosing was significantly higher than in the twice-daily dosing group. ${ }^{11}$ The present study demonstrated that the efficacy of multimatrix mesalazine $2.4 \mathrm{~g}$ /day QD in the maintenance of the absence of rectal bleeding was noninferior to that of multiple-dose formulation of mesalazine, Time- $2.25 \mathrm{~g} /$ day TID, with a comparable safety profile. This is aligned with the European Crohn's and Colitis Organisation consensus that once-daily administration of 5-ASA is at least as effective as twice- or 3 times-daily administration, with no increased side effects, in the maintenance of remission of UC. ${ }^{12}$

This study adopted rectal bleeding as the primary endpoint, and the proportion of patients who maintained a rectal bleeding score of 0 for 48 weeks was $84.8 \%$ in the Multimatrix-2.4 g/day group and $78.0 \%$ in the Time- $2.25 \mathrm{~g} /$ day group. Similar results were obtained in a previous noninferiority study of pH-dependent delayed-release mesalazine $2.4 \mathrm{~g} /$ day TID, which also assessed rectal bleeding as the primary endpoint compared with controlled-release mesalazine $2.25 \mathrm{~g} /$ day TID. ${ }^{13}$ The proportion of patients without rectal bleeding for 48 weeks was $76.9 \%$ in the $\mathrm{pH}$-dependent delayed-release mesalazine $2.4 \mathrm{~g}$ /day TID group and $69.2 \%$ in the controlled-release mesalazine $2.25 \mathrm{~g}$ /day TID group. In another phase IIIb/IV open-label study of multimatrix mesalazine (the MOMENTUM trial), rectal bleeding was also investigated as one of the secondary endpoints during maintenance treatment with multimatrix mesalazine $2.4 \mathrm{~g} /$ day. ${ }^{14}$ The proportion of patients who maintained a rectal bleeding score of 0 for 12 months was $65.4 \%$ in patients 
with complete remission (modified UC-DAI score $\leq 1$ with a score of 0 for both rectal bleeding and stool frequency, and $\geq 1$-point reduction in endoscopy score from baseline) after induction treatment with multimatrix mesalazine $4.8 \mathrm{~g} /$ day for 8 weeks.

The results for the secondary endpoints (duration of nonoccurrence of rectal bleeding, relapse, change in the UC-DAI score, and change in the UC-DAI subscores) supported the results of the primary endpoint. In the subgroup analysis, Multimatrix-2.4 g/day QD demonstrated consistent efficacy results regardless of disease type, extent of lesion, and baseline UC-DAI score. The safety was comparable between the 2 treatment groups.

Since this study adopted a double-dummy design, the compliance with both multimatrix mesalazine tablets and controlled-release mesalazine tablets was measured in all patients. In this well-controlled study, the mean rates of compliance with each drug in all analysis sets were not less than $95 \%$ in both treatment groups. Although this situation does not reflect clinical practice, a difference in compliance between QD dosing and TID dosing was not detected in this study.

In conclusion, this study showed that QD multimatrix mesalazine administered as 2 tablets achieved a remission maintenance effect that was not inferior to a controlled-release mesalazine $2.25 \mathrm{~g} /$ day formulation administered TID.

\section{ACKNOWLEDGEMENTS}

The authors would like to thank all patients and the following investigators who participated in this study. The affiliation of each investigator provided here was correct at the time the study was conducted.

Investigators: Satoshi Motoya, Sapporo-Kosei General Hospital; Tomohiro Tada, Tada Tomohiro Clinic; Toshihide Ohmori, Ohmori Toshihide Gastro-intestinal Clinic; Takehiro Arai, Tokatsu-Tsujinaka Hospital; Yukihiro Hamahata, Tsujinaka Hospital Kashiwanoha; Yasuo Suzuki, Toho University Sakura Medical Center; Toshiaki Terada, Terada Hospital; Jun Miwa, Toshiba General Hospital; Takashi Hiratsuka, Hiratsuka Gastroenterological Hospital; Yasuyoshi Takeo, Hachioji Digestive Disease Hospital; Toru Isono, Isono Clinic; Ryuzo Murai, Onaka Clinic; Nobuyuki Matsuhashi, NTT Medical Center Tokyo; Shinichiro Suzuki, Fujisawa Shounandai Hospital; Koichiro Matsuda, Toyama Prefectural Central Hospital; Kazuhiko Kawakami, Matsuda Hospital; Tadashi Yokoyama, Yokoyama Gastrointestinal Hospital; Mitsuki Miyata, Miyata Clinic; Akihiko Ohta, Ieda Hospital;
Takayuki Yamamoto, Yokkaichi Social Insurance Hospital; Takataro Fukuhara, Hirakata Kohsai Hospital; Soken Sai, Sai Clinic; Tatsuhiko Usui, Usui Internal Medicine/Gastroenterology Department Clinic; Kentaro Tsuji, Tsuji Kentaro Clinic; Koji Sawada, Ikoma Digestive Tract Internal Medicine Clinic; Kenji Watanabe, Osaka City University Hospital; Kazuhiko Nishimura, Nishimura Internal Medicine Clinic; Nobuo Aoyama, Aoyama Medical Clinic; Shiro Nakamura, Ken Fukunaga, Hyogo College of Medicine Hospital; Akira Chikuba, Chikuba Hospital for Gastrointestinal and Colorectal Surgery; Toshiaki Ochiai, Teppei Kabemura, Saiseikai Fukuoka General Hospital; Hisamitsu Hidaka, Hidaka Clinic; Tetsuya Ishida, Oita Red Cross Hospital; Naoto Ishikawa, Ishikawa Clinic; Naoya Shibata, Michihisa Fuchimoto, Ikime Coloproctological Clinic; Yukinori Sameshima, Sameshima Hospital; Hirokazu Oshimoto, Isesaki Municipal Hospital; Naoki Yoshimura, Social Insurance Central General Hospital; Takafumi Ando, Nagoya University Hospital; Takashi Hisabe, Fukuoka University Chikushi Hospital; Shujiro Yazumi, Kitano Hospital; Tsuguhiro Kimura, Fujita Gastroenterological Division Hospital; Shinichi Ogata, Saga Prefectural Medical Centre Koseikan; Hirozumi Obata, Obata Medical Clinic.

Multimatrix mesalazine was kindly provided by Shire US Inc., Wayne, PA, USA and controlled-release mesalazine was kindly provided by Kyorin Pharmaceutical Co., Ltd, Tokyo, Japan. We would also like to thank Shire US Inc. and Kyorin Pharmaceutical Co., Ltd.

\section{REFERENCES}

1. Miner P, Hanauer S, Robinson M, Schwartz J, Arora S. Safety and efficacy of controlled-release mesalamine for maintenance of remission in ulcerative colitis: Pentasa UC Maintenance Study Group. Dig Dis Sci 1995;40:296-304.

2. Ardizzone S, Petrillo M, Imbesi V, Cerutti R, Bollani S, Bianchi Porro G. Is maintenance therapy always necessary for patients with ulcerative colitis in remission? Aliment Pharmacol Ther 1999;13:373-379.

3. Hanauer SB, Sninsky CA, Robinson M, et al. An oral preparation of mesalamine as long-term maintenance therapy for ulcerative colitis: a randomized, placebo-controlled trial. Ann Intern Med 1996;124:204-211.

4. Hawkey CJ, Dube LM, Rountree LV, Linnen PJ, Lancaster JF. A trial of zileuton versus mesalazine or placebo in the maintenance of remission of ulcerative colitis: the European Zileuton Study Group for Ulcerative Colitis. Gastroenterology 1997;112: 718-724. 
5. Feagan BG, Macdonald JK. Oral 5-aminosalicylic acid for maintenance of remission in ulcerative colitis. Cochrane Database Syst Rev 2012;10:CD000544. doi: 10.1002/14651858.CD000544. pub3.

6. D’Haens G, Sandborn WJ, Barrett K, Hodgson I, Streck P. Oncedaily MMX( $(\circledR)$ mesalamine for endoscopic maintenance of remission of ulcerative colitis. Am J Gastroenterol 2012;107:10641077.

7. Prantera C, Kohn A, Campieri M, et al. Clinical trial: ulcerative colitis maintenance treatment with 5-ASA: a 1-year, randomized multicentre study comparing MMX with Asacol. Aliment Pharmacol Ther 2009;30:908-918.

8. Sutherland LR, Martin F, Greer S, et al. 5-Aminosalicylic acid enema in the treatment of distal ulcerative colitis, proctosigmoiditis, and proctitis. Gastroenterology 1987;92:1894-1898.

9. Watanabe M, Hanai H, Nishino H, Yokoyama T, Terada T, Suzuki Y. Comparison of QD and TID oral mesalazine for maintenance of remission in quiescent ulcerative colitis: a doubleblind, double-dummy, randomized multicenter study. Inflamm Bowel Dis 2013;19:1681-1690.
10. Sandborn WJ, Korzenik J, Lashner B, et al. Once-daily dosing of delayed-release oral mesalamine (400-mg tablet) is as effective as twice-daily dosing for maintenance of remission of ulcerative colitis. Gastroenterology 2010;138:1286-1296.e3.

11. Dignass AU, Bokemeyer B, Adamek H, et al. Mesalamine once daily is more effective than twice daily in patients with quiescent ulcerative colitis. Clin Gastroenterol Hepatol 2009;7:762769.

12. Dignass A, Lindsay JO, Sturm A, et al. Second European evidence-based consensus on the diagnosis and management of ulcerative colitis part 2: current management. J Crohns Colitis 2012;6:991-1030.

13. Ito H, Iida M, Matsumoto T, et al. Direct comparison of two different mesalamine formulations for the maintenance of remission in patients with ulcerative colitis: a double-blind, randomized study. Inflamm Bowel Dis 2010;16:1575-1582.

14. Rubin DT, Bradette M, Gabalec L, et al. Ulcerative colitis remission status after induction with mesalazine predicts maintenance outcomes: the MOMENTUM Trial. J Crohns Colitis 2016; 10:925-933. 